\title{
Robust Deconvolution of High-Frequency Ultrasound Images Using Higher-Order Spectral Analysis and Wavelets
}

\author{
Suiren Wan, Balasundar I. Raju, and Mandayam A. Srinivasan
}

\begin{abstract}
Deconvolution of high-frequency (30-40 MHz) ultrasonic images of human skin was studied in vivo. Separate one-dimensional (1-D) functions for the axial and lateral profiles were first estimated using higher-order spectral methods. Subsequently, deconvolution was implemented using a regularized inverse Wiener filtering of the wavelet and scaling coefficients that were obtained after a wavelet decomposition of the RF signals. Deconvolution was first performed in the axial direction, then in the lateral direction. The methods were applied to data obtained from the skin of 16 volunteers using three different transducers. Significant improvements in both the axial and lateral resolutions were obtained in all the cases. Features such as hair follicles in the dermis and fingerprints on the surface of the finger were more clearly displayed in the processed images compared to the original images. The results indicate that the deconvolution method using higher-order spectral methods and wavelet analysis could significantly improve the quality of high-frequency ultrasonic skin images.
\end{abstract}

\section{INTRODUCTION}

$\mathrm{U}$ LTRASOUND images are blurred in both the axial and lateral directions due to the finite resolution of the imaging system. A convenient model to represent this blurring is to express each echo line signal $y(n)$ (RF signal corresponding to one A-scan) as a convolution of a system function $h(n)$ with a tissue reflectivity function $x(n)$ :

$$
y(n) \doteq h(n)^{*} x(n)+\eta(n)
$$

where $\eta(n)$ is an additive noise term. A similar expression also can be written for the lateral smearing due to the finite beam width. Deconvolution, the process of recovering the original $x(n)$ from measurements of $y(n)$, not only serves to improve the resolution of the images, but also tends to make the appearance of the images uniform across many subjects by removing system-dependent effects. Several authors [1]-[5] have studied the deconvolution process applied to ultrasound imaging. In most of the

Manuscript received December 8, 2002: accepted March 18, 2003 The work reported here was supported by NIH grant NS33778, a Grant-in-Aid of Research from the National Academy of Sciences through Sigma Xi, and by Hwa-Ying Culture and Education Foundation.

The authors are with the Laboratory for Human and Machine Haptics, Research Laboratory of Electronics, Massachusetts Institute of Technology, Cambridge, MA 02139.

B. I. Raju's current address is Philips Research, 345 Scarborough Road, Briarcliff Manor, NY 10510 (e-mail: raju@alum.mit.edı). methods, the system function $h(n)$ is often considered unknown and is estimated during the deconvolution process itself. Typically, the signals $x(n)$ and $h(n)$ are separated in the cepstrum domain. The cepstrum of a signal is defined as the inverse Fourier transform of the logarithm of the Fourier transform of that signal. The basic assumption in this method is that the two signals have different cepstral signatures that can be clearly distinguished. AIternate definitions of the cepstrum such as the square root; cepstrum and generalized cepstrum also have been shown to be useful for deconvolution [6]. The limitation of this approach is that it is hard to separate the two signals $x(n)$ and $h(n)$ in the cepstral domain because a simple? "cut-off" to separate the two may not exist. For instance, there is no a priori reason to believe that the cepstrum of $h(n)$ is limited to the first few terms and that of the signal $x(n)$ occupies the higher terms. When a cut-off is used to separate the two, the estimated $h(n)$ inherently contains the effects of $x(n)$. Another approach to deconvolution uses the cepstrum of not $y(n)$, but rather of the higher order cumulants of $y(n)$ [3], [7]. This method is applicable only for non-Gaussian tissue signals $x(n)$. If the assumption of non-Gaussianity is satisfied, this method offers many advantages over the previous method. The separation of the two signals $h(n)$ and $x(n)$ is straightforward, and no assumptions regarding the cepstral signatures of $h(n)$ and $x(n)$ are needed. Also, the deconvolution process is immune to additive Gaussian noise, as a Gaussian signal has zero cumulants of order greater than two [8].

Our primary interest in this work is in improving the image quality of high-frequency ultrasound $(30-40 \mathrm{MHz})$ images of skin tissues. Due to finite resolution, features such as the fingerprints, sweat glands, hair follicles, and so forth often appear blurred in ultrasound images of the skir. Although the blurring can be reduced by using very high frequency $(100 \mathrm{MHz})$ ultrasound [9], the depth of imaging at these frequencies is severely limited due to the increased attenuation. The relevant ultrasonic frequency for most clinical dermatological applications is in the range $30-40 \mathrm{MHz}$. Hence, deconvolution tools are important in improving image quality of skin images. In our previous study, we showed that the envelope signals from skin tissues are non-Rayleigh distributed when data were collected using transducers that are typically used in skin imaging [10]. This implies that the corresponding RF signals are non-Gaussian. Because of this, the cepstrum method, 
based on higher-order cumulants, is of particular importance, and therefore, it is used in this work.

In practice, the deconvolution process usually is accomplished in two steps. In the first step, the system function is estimated using the measured signal $y(n)$ using one of several cepstrum-based methods. In the next step, the tissue signal $x(n)$ is estimated through appropriate regularized inverse methods. Commonly, the Wiener filter is used to recover $x(n)$ from noisy measurements of the signal $y(n)$. However, the Wiener filter implemented in the Fourier domain is not well suited for representing spatially localized phenomena such as the edges, ridges, and sharp boundaries of images because the Fourier basis functions lack localization in time or space. This usually presents as a Gibbs phenomenon on edges and boundaries on which the ringing distorts the localized features. This problem can be avoided through the use of wavelets that have localization in both time and frequency (scale). Because wavelets are scaled waveforms, by moving through scale, various image structures such as ridges and boundaries in fingertip skin can be selectively characterized. Unlike Fourier methods that rely on a single basis $\exp (j \omega n)$, wavelets provide flexibility in the choice of the basis function that can be selected to match the transmitted pulse. An additional advantage with wavelet analysis is that the signals can be sparsely represented with a few nonzero coefficients, leading to efficient methods for tasks such as compression and denoising. Hence, in this work, a regularized deconvolution technique based on wavelets, which has been proposed by Neelamani et al. [11] is used to implement the deconvolution of high-frequency ultrasound images of the skin once $h(n)$ is estimated using higher-order spectral methods.

This paper is organized as follows. In Section II, the process of estimating the system function using higher order cumulants is described. In Section III, the waveletbased regularized deconvolution method is presented. In Section IV, experiments and results on deconvolution applied to high-frequency ultrasound images of the skin are described. The axial and lateral deconvolution steps were assumed to be separable for the sake of simplicity. Because the process of image formation actually occurs in the RF domain rather than the envelope domain, the deconvolution procedure is implemented first in the axial direction, then in the lateral direction. Section $V$ concludes the paper with a discussion of the results.

\section{Estimation of System Function Using Higher-Order SPECTRAl ANalysis}

The system functions in both the axial and lateral directions were obtained using higher-order spectral methods. We use the notation $h(n)$ to represent both the axial and lateral system functions without loss of generality. In (1), $h(n)$ is a deterministic, causal, FIR filter of length $\mathrm{M}, x(n)$ is the tissue response function, which is assumed to be stationary, white, zero mean and non-Gaussian, and $\eta(n)$ is a zero mean Gaussian noise that is independent of $x(n)$.
The third-order cumulant of the zero mean signal $y(n)$ is given by [8], [12]:

$$
\begin{aligned}
c_{y}\left(m_{1}, m_{2}\right) & =E\left[y(n), y\left(n+m_{1}\right), y\left(n+m_{2}\right)\right] \\
& =\gamma_{x} \frac{1}{M} \sum_{k=0}^{M-1} h(k) h\left(k+m_{1}\right) h\left(k+m_{2}\right),
\end{aligned}
$$

where $\gamma_{x}=E\left[x^{3}(n)\right]$ is a constant equal to the third cumulant of the zero mean signal $x(n)$, which is assumed to be nonzero. The expression $E[$.$] represents statistical$ average. The bispectrum $C_{y}\left(z_{1}, z_{2}\right)$ is defined as the 2-D Z-transform of the third-order cumulant:

$$
C_{y}\left(z_{1}, z_{2}\right)=\sum_{m_{1}} \sum_{m_{2}} c_{y}\left(m_{1}, m_{2}\right) z_{1}^{-m_{1}} z_{2}^{-m_{2}} .
$$

From (2) and (3) we get:

$$
C_{y}\left(z_{1}, z_{2}\right)=\gamma_{x} H\left(z_{1}\right) H\left(z_{2}\right) H\left(z_{1}^{-1} z_{2}^{-1}\right) .
$$

The bicepstrum of the third-order cumulant is defined as the inverse 2-D z-transform of the logarithm of the bispectrum:

$$
b_{y}\left(m_{1}, m_{2}\right)=Z^{-1}\left[\ln \left(C_{y}\left(z_{1}, z_{2}\right)\right)\right] .
$$

The bicepstrum $b_{y}\left(m_{1}, m_{2}\right)$ can be computed using the following expression [13]:

$$
b_{y}\left(m_{1}, m_{2}\right)=\frac{1}{m_{1}} F^{-1}\left\{\frac{F\left[m_{1} c_{y}\left(m_{1}, m_{2}\right)\right]}{F\left[c_{y}\left(m_{1}, m_{2}\right)\right]}\right\},
$$

where $F$ and $F^{-1}$ represent 2-D fast Fourier transform (FFT) and its inverse, respectively.

In order to relate the bicepstrum to the system function, consider the logarithm of the bispectrum in (4):

$$
\begin{aligned}
\ln \left(C_{y}\left(z_{1}, z_{2}\right)\right)=\ln \left(\gamma_{x}\right)+\ln \left(H\left(z_{1}\right)\right) & \\
& +\ln \left(H\left(z_{2}\right)\right)+\ln \left(H\left(z_{1}^{-1} z_{2}^{-1}\right)\right) .
\end{aligned}
$$

Denoting the cepstrum of $h(n)$ as $\hat{h}(n)$ and combining (5) and (7), we have

$$
\begin{aligned}
& \sum_{m_{1}} \sum_{m_{2}} b_{y}\left(m_{1}, m_{2}\right) z_{1}^{-m_{1}} z_{2}^{-m_{2}}=\ln \left(\gamma_{x}\right)+\sum_{m_{1}} \hat{h}\left(m_{1}\right) z_{1}^{-m_{1}} \\
& \quad+\sum_{m_{1}} \hat{h}\left(m_{2}\right) z_{2}^{-m_{2}}+\sum_{m_{1}, m_{2}=m_{1}} \hat{h}\left(m_{1}\right) z_{1}^{m_{1}} z_{2}^{m_{2}} \\
& \quad=\sum_{m_{1}} \sum_{m_{2}}\left[\ln \left(\gamma_{x}\right) \delta\left(m_{1}\right) \delta\left(m_{2}\right)+\hat{h}\left(m_{2}\right) \delta\left(m_{2}\right)\right. \\
& \left.+\hat{h}\left(m_{2}\right) \delta\left(m_{1}\right)+\hat{h}\left(-m_{1}\right) \delta\left(m_{1}-m_{2}\right)\right] z_{1}^{-m_{1}} z_{2}^{-m_{2}} .
\end{aligned}
$$

Thus $\hat{h}(n)$ can be obtained for all $n \neq 0$ by evaluating the bicepstrum along the diagonal $m_{1}=m_{2}$ :

$$
\hat{h}(n)=b_{y}(-n,-n) \quad n \neq 0 .
$$

The value of $\hat{h}(0)$ cannot be determined as $\gamma_{x}$ is unknown, but its exact value is not needed as a different $\hat{h}(0)$ only 
results in a different overall scaling for the pulse $h(n)$. We get the estimated $h(n)$ :

$$
h(n)=Z^{-1}\{\exp [Z(\hat{h}(n))]\}
$$

where the symbols $Z($.$) and Z^{-1}($.$) represent the Z$ transform and its inverse, respectively. To summarize, we first compute the bicepstrum $b_{y}\left(m_{1}, m_{2}\right)$ of the recorded signal, evaluate it along the diagonal to obtain $\hat{h}(n)$ (with an appropriate normalization determined by the choice of $\hat{h}(0)$ ), and compute the inverse cepstrum to obtain the system function $h(n)$. It is worth mentioning that $h(n)$ and $x(n)$ are decoupled in (4), as the effect of $x(n)$ appears only as a multiplicative constant. This means that, for non-Gaussian tissue signals with nonzero third cumulants, the estimated system function $h(n)$ is theoretically independent of the tissue being used to determine it. As demonstrated by our results, $h(n)$ estimated from only one subject was subsequently used for deconvolution of data from all subjects. This is generally not possible with firstorder cepstral methods.

\section{Wavelet Deconvolution Method}

\section{A. Discrete Wavelet Transform}

A signal of length $2^{N}$, can be represented in the wavelet domain as follows:

$$
y(n)=\sum_{j=1}^{J} \sum_{k=1}^{2^{(N-j)}} d_{j k} \psi_{j k}(n)+\sum_{k=1}^{2^{(N-J)}} a_{J k} \phi_{J k}(n), \quad J \leq N
$$

where $J$ is the number of levels in the analysis of the function $y(n), d_{j k} \psi_{j k}(n)$ is referred to as the detail component of $y(n)$ at scale $j$ and location $k$, and $a_{J k} \phi_{J k}(n)$ is referred to as an approximation of $y(n)$ at scale $J$ and location $k$. The functions $\psi_{j k}(n)$ and $\phi_{J k}(n)$ are described as follows:

$$
\begin{aligned}
& \psi_{j k}(n)=2^{-j / 2} \psi\left(2^{-j} n-k+1\right) \\
& 1 \leq j \leq J, \quad 1 \leq k \leq 2^{N-j} \\
& \phi_{J k}(n)=2^{-J / 2} \phi\left(2^{-J} n-k+1\right) \\
& 1 \leq k \leq 2^{N-J}
\end{aligned}
$$

where $\psi(n)$ is the wavelet function and $\phi(n)$ is the scaling function. The coefficients $d_{j k}$ and $a_{J k}$ are defined as the inner products between $y(n)$ and the wavelet and scaling functions:

$$
\begin{aligned}
& d_{j k}=\left\langle y, \psi_{j k}\right\rangle, \\
& a_{j k}=\left\langle y, \phi_{j k}\right\rangle .
\end{aligned}
$$

In practice, the above coefficients are computed efficiently using a cascade algorithm that is faster than the computation of FFTs [14]. Any signal processing task on the wavelet domain (e.g., denoising) can be thought of as
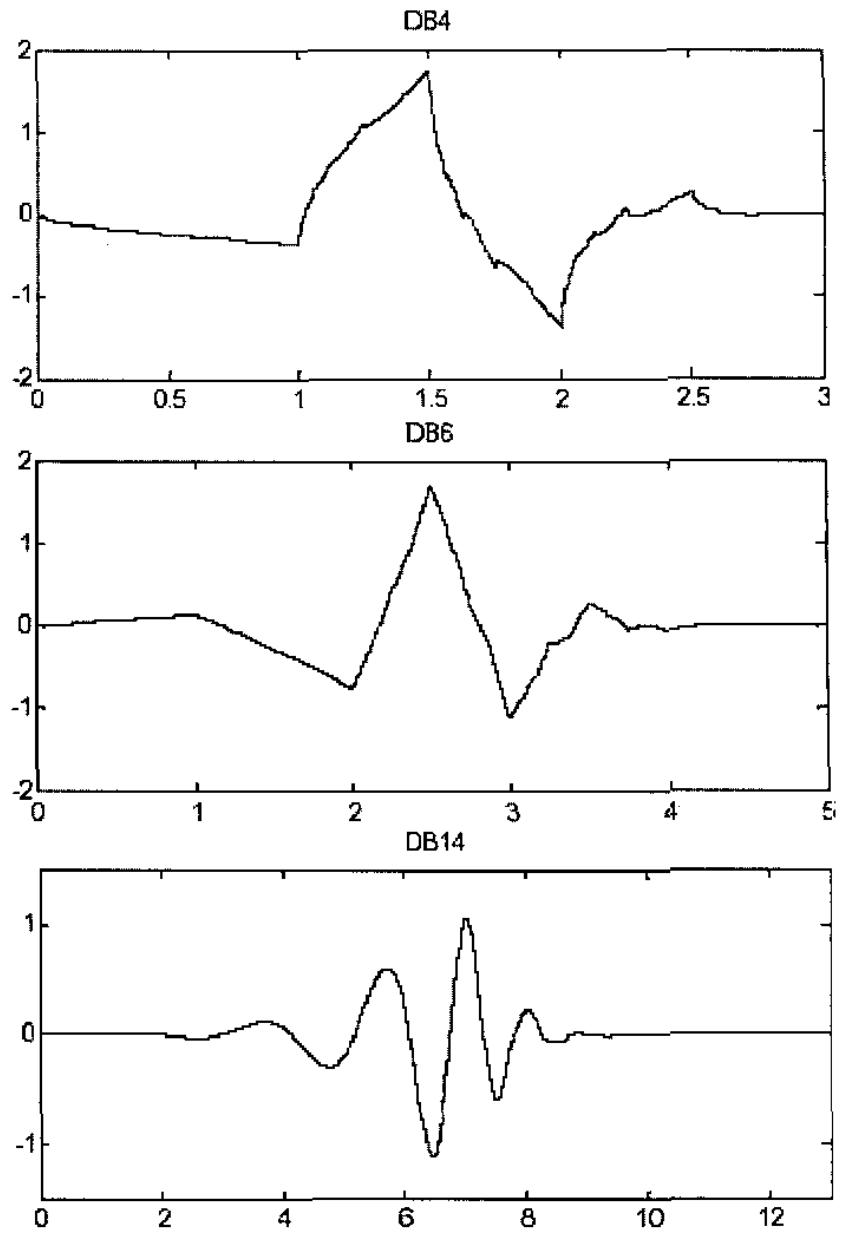

Fig. 1. The Daubechies wavelets used in this work. The DB14 wavelet is similar to the reflection from a plane reflector placed at the focus. The DB6 wavelet is roughly similar to the lateral profile of the transducer. It should be noted that the scale on the time axis is differen: for the three wavelets, with DB4 having the smallest time support.

suitable manipulations of the coefficients $d_{j k}$ and $a_{J k}$. The simplest rule for selecting a suitable wavelet is to choose one that has a shape similar to that of the processed function so that the decomposition of the function is highly efficient with fewer nonzero coefficients. Another factor is the length of the time support allowed for the wavelet. In this work, different wavelets all belonging to the Daubechies family, were used at various stages in the deconvolution process. These wavelets are denoted as $\operatorname{DB} n$ where $n$ is the order and in our convention always even (hence $n=2$ corresponds to the Haar wavelets). The three Daubechies wavelets used in this study are shown in Fig. 1. As described later, the DB14 and DB6 wavelets were used in the denoising stage of the axial and lateral deconvolution processes, respectively. The DB4 wavelets were used in thin the wavelet-Wiener filtering stage for both the axial and lateral deconvolution stages. Additionally, the DB14 wavelet also was used for a predenoising stage before attenuation and diffraction compensation. These steps are described in detail below. 


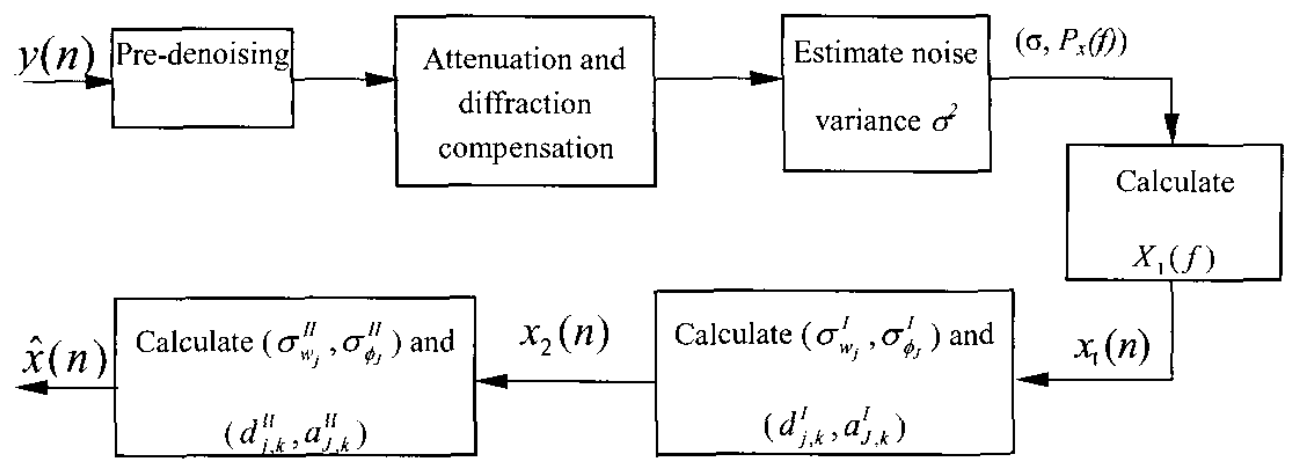

Fig. 2. Block diagram of the wavelet deconvolution method applicd in the axial direction. The lateral deconvolution is similar but does not contain the predenoising and attenuation and diffraction compensation stages.

\section{B. Overall Method}

In this section, a brief summary of the overall procedure is presented. A block diagram of the axial deconvolution process is shown in Fig. 2. The system function is assumed to be estimated already using higher-order spectral methods at the beginning of the process. Because deconvolution techniques work better when the signal-to-noise ratio (SNR) is high, a prenoising procedure is first applied to the RF data. In the case of high-frequency skin imaging, a noncontact transducer placed above the skin surface is used, because of which the recorded RF data contains signals from the coupling medium (water) which is essentially receiver noise. The predenoising procedure serves to minimize this noise so that diffraction compensation easily could be applied to the data. Otherwise, after compensation, any noise present at regions away from the focus would be amplified. After predenoising; compensation for both attenuation and diffraction is applicd. After compensation, a two-stage deconvolution method is applied. The first stage consists of the usual regularized inverse Wiener filtering that gives a preliminary estimate of the tissue function denoted as $x_{1}(n)$. The second stage consists of a wavelet domain denoising together with Wiener filtering of the wavelet coefficients that gives the final estimate $\hat{x}(n)$. Once the axial deconvolution is done, the lateral deconvolution is done on the axially deconvolved RF data in a similar manner, but without the predenoising and attenuation and diffraction compensation stages.

\section{Predenoising Using Wavelets}

The recorded RF signals were denoised using a threshold method [14], [15] that set some of the finest scale wavelet coefficients to zero. Two kinds of throsholding commonly used are the soft and hard thresholding methods. In our work, no apparent difference was found between the two, and the hard thresholding was used:

$$
d_{1 k}= \begin{cases}d_{1 k} & \text { if }\left|d_{1 k}\right|>T \\ 0, & \text { if }\left|d_{1 k}\right| \leq T\end{cases}
$$

The threshold value was chosen to be equal to $T=$ $\sigma \sqrt{2 \log _{e} L}$ where $L$ is the length of the RF signal and $\sigma$ is the noise standard deviation, which was taken to be 1.5 times the median of the finest scale wavelet coefficients. The DB14 wavelets were used in this step.

\section{Attenuation Compensation}

The deconvolution process requires the backscattered signals from a particular tissue to be spatially invariant with respect to depth. One effect that causes the echo signals from the same tissue to change with depth is attenuation. To compensate for attenuation, a previously determined attenuation coefficient of $0.2 \mathrm{~dB} / \mathrm{mm} / \mathrm{MHz}$ for skin was used [16]. Attenuation compensation was applied to the RF echo signals at the transducer's center frequency.

\section{E. Diffraction Compensation}

Another effect that causes the echo signals to change with depth is diffraction due to the use of focused transducers. This effect is frequency dependent, with higher frequencies being more tightly focused and showing a larger variation with depth. To compensate for this effect, analytical expressions developed by Chen et al. [17] were used to compensate the echo signals. We also had confirmed in a previous work that these analytical expressions were reasonably accurate [16]. The diffraction compensation curves were computed at the transducer's center frequency.

\section{F. Deconvolution with Wavelets}

Deconvolution methods commonly require regularization techniques due to the presence of noise as well as zeros in the system transfer function. The regularized Wiencr inverse filter is one such example. However, the Wiener filter is commonly implemented in the Fourier domain and is not well suited for signals that contain localized phenomena such as edges. These features are distorted during the processing; leading to ringing effects. The use of wavelet transforms overcomes this problem due to the ability of wavelets to capture localized phenomena. The waveletvaguelette decomposition (WVD) method developed by 
Donoho [18], performs a simple inversion in the Fourier domain using $H^{-1}(f)$ to obtain a noisy, unbiased estimate of the input, followed by "wavelet shrinkage" in which some of the wavelet coefficients are reduced depending on the variance of the noise. The WVD method has the drawback in that the noise variance becomes large when the system function contains zeros, making the method ill-posed. A hybrid method combining the regularized Wiener filter in the Fourier domain and the WVD approach, was proposed by Neelamani et al. [11] to overcome this limitation. In the present work, we have adapted this method for deconvolution of high-frequency ultrasound data. This method is robust due to the combination of the regularized Wiener filter that can handle zeros in the system transfer function and the wavelet method that avoids the ringing artifacts. The procedure consists of two steps:

1. Regularized Inverse Wiener Filtering: An initial estimate of $x(n)$ denoted as $x_{1}(n)$ is first obtained using the regularized Wiener inverse filter $W(f)$ :

$$
\left\{\begin{array}{l}
X_{1}(f)=W(f) Y(f) \\
W(f)=\frac{H^{*}(f) P_{x_{1}}(f)}{|H(f)|^{2} P_{x_{1}}(f)+\alpha \sigma^{2}}
\end{array}\right.
$$

where $X_{1}(f)$ and $Y(f)$ are the Fourier transforms of $x_{1}(n)$ and $y(n)$, respectively; $P_{x_{1}}(f)$ is the power density spectrum of $x_{1}(n) ; \alpha$ is regularization parameter; and $\sigma^{2}$ is the noise variance. Because $P_{x_{1}}(f)$ is unknown to begin with, it was estimated with using an iterative Wiener method [19]. A value of $\alpha=0.1$ was found to be suitable after a few trials. The noise variance $\sigma^{2}$ was calculated as the median of the finest scale wavelet coefficients as described by Donoho and Johnstone [15].

2. Wavelet Shrinkage and Wavelet-Based Wiener Filtering: Due to limitations of Fourier domain processing, $x_{1}(n)$ has distortions due to the presence of edges and boundaries. In order to improve the signal, $x_{1}(n)$ is decomposed on the wavelet domain and a denoising of the wavelet coefficients is performed:

$$
x_{2}(n)=\sum_{j=1}^{J} \sum_{k=1}^{2^{N-j}} d_{j k}^{I} \psi_{j k}^{I}(n)+\sum_{k=1}^{2^{(N-J)}} a_{J k}^{I} \phi_{J k}^{I}(n), \quad J \leq N,
$$

where $d_{j k}^{I}$ and $a_{J k}^{I}$ are the wavelet and scaling coefficients after the application of the denoising threshold:

$$
\begin{aligned}
& d_{j k}^{I}=\left\{\begin{array}{ll}
\left\langle x_{1}, \psi_{j k}^{I}\right\rangle & \text { if }\left|\left\langle x_{1}, \psi_{j k}^{I}\right\rangle\right|>\sigma_{\psi_{j}}^{I}, \\
0 & \text { if }\left|\left\langle x_{1}, \psi_{j k}^{I}\right\rangle\right| \leq \sigma_{\psi_{j}}^{I}
\end{array},\right. \\
& a_{j k}^{I}= \begin{cases}\left\langle x_{1}, \phi_{J k}^{I}\right\rangle & \text { if }\left|\left\langle x_{1}, \phi_{J k}^{I}\right\rangle\right|>\sigma_{\phi_{j}}^{I}, \\
0 & \text { if }\left|\left\langle x_{1}, \phi_{J k}^{I}\right\rangle\right| \leq \sigma_{\phi_{j}}^{I}\end{cases}
\end{aligned}
$$

The DB14 and DB6 functions were used in this step, depending on whether axial or lateral deconvolution is being performed, respectively. The superscript $I$ is used to distinguish the wavelets and coefficients from another set of wavelets and cocfficients mentioned below. The number of levels $J$ was three. It should be noted that, in general, the threshold parameters depend on the scale and are different for the wavelet and scaling coefficients. Therefore, estimates of the noise at various scales were obtained using the median of the cocfficients at each scale.

After the signals were denoised, the signal estimate $\hat{x}(n)$ was obtained using Wiener filters in the wavelet domain: where the filtering is done for the wavelet coefficients [20]:

$$
\hat{x}(n)=\sum_{j=1}^{J} \sum_{k=1}^{2^{N-j}} \hat{d}_{j k}^{I I} \psi_{j k}^{I I}(n)+\sum_{k=1}^{2^{N-J}} \hat{a}_{J k}^{I I} \phi_{J k}^{I I}(n), \quad J \leq N
$$

where:

$$
\begin{aligned}
& \hat{d}_{j k}^{I I}=\frac{\left|d_{j k}^{I I}\right|^{2}\left\langle x_{1}, \psi_{j k}^{I I}\right\rangle}{\left|d_{j k}^{I I}\right|^{2}+\left|\sigma_{\psi_{j}}^{I I}\right|^{2}}, \quad \text { and } \begin{array}{l}
d_{j k}^{I I}=\left\langle x_{2}, \psi_{j k}^{I I}\right\rangle \\
\hat{a}_{J k}^{I I}=\frac{\left|a_{J k}^{I I}\right|^{2}\left\langle x_{1}, \phi_{J k}^{I I}\right\rangle}{\left|a_{J k}^{I I}\right|^{2}+\left|\sigma_{\phi_{J}}^{I I}\right|^{2}},
\end{array}
\end{aligned}
$$

The DB4 wavelet and scaling functions were used for this last step for both the axial and lateral deconvolution steps. In general, a wavelet representation with a longer time support is preferred for denoising, and a smaller time: support such as DB4 is preferred for the final Wiener fil.. tering.

\section{EXPERIMENTS AND RESULTS}

\section{A. Experimental System}

The experimental system consisted of a mechanically scanned, single-element PVDF transducer (Panametrics, Waltham, MA), a pulser/receiver (Panamet.. rics; Model 5900), a three-axis scanning system (ParkerHannifin/Compumotor, Cleveland, $\mathrm{OH}$ ), a high-speed A2D board (Gage Applied Sciences, Montreal, Canada), and a personal computer to control mechanical scanning and data acquisition. Three transducers were used in this study in order to demonstrate robustness of the methods under different systems. The specifications of these transducers are shown in Table I. The transducers were mounted on the three-axis stage that had encoders on the $\mathrm{x}$ - and $\mathrm{y}$-axis that provided a high lateral positioning accuracy of $1 \mu \mathrm{m}$. The backscattered signals were sampled at a sampling frequency of $200 \mathrm{MHz}$. The spacing between two adjacent echo lines was $25 \mu \mathrm{m}$ for transducers I and II, and $15 \mu \mathrm{m}$ for transducer III. Water was used as a couplingr medium between the transducers and tissues.

\section{B. Human Subjects and Tissues}

A total of 16 subjects, 8 male and 8 female, were used in this study. Table II describes the subject population, the number of data sets collected for each transducer, and the body sites imaged. More data were collected from transducer I as this transducer provided the optimum compromise between penetration depth and resolution needed for: 
TABLE I

'Transducers Used in This Study. ${ }^{1}$

\begin{tabular}{cccc}
\hline Transducer & $\begin{array}{c}\text { I } \\
\text { (Model PI50) }\end{array}$ & $\begin{array}{c}\text { II } \\
\text { (Model PI75) }\end{array}$ & $\begin{array}{c}\text { III } \\
\text { (Model PI3005) }\end{array}$ \\
\hline Center frequency & $33 \mathrm{MHz}$ & $30 \mathrm{MHz}$ & $44 \mathrm{MHz}$ \\
-6 dB bandwidth & $28 \mathrm{MHz}$ & $40 \mathrm{MHz}$ & $50 \mathrm{MHz}$ \\
F-number & 2 & 4 & 2 \\
Focal length & $12.7 \mathrm{~mm}$ & $12.7 \mathrm{~mm}$ & $: 4 \mathrm{~mm}$ \\
Diameter & $6.35 \mathrm{~mm}$ & $3.175 \mathrm{~mm}$ & $2 \mathrm{~mm}$ \\
Axial resolution & $27 \mu \mathrm{m}$ & $19 \mu \mathrm{m}$ & $15 \mu \mathrm{m}$ \\
Lateral resolution & $83 \mu \mathrm{m}$ & $200 \mu \mathrm{m}$ & $68 \mu \mathrm{m}$ \\
\hline
\end{tabular}

${ }^{1}$ The axial and lateral resolutions were computed based on theoretical $-6 \mathrm{~dB}$ beam widths. Axial resolution $=\frac{c}{2 \times B W}$ where $c$ is the speed of sound, and $B W$ is the $-6 \mathrm{~dB}$ bandwidth of the signal reflected from a planar reflector at the focus. Lateral resolution $=F n u m \times \lambda$ where Fnum is the f-number of the transducer specified by the manufacturer and $\lambda$ is the wavelength corresponding to the center frequency of the signal reflected from a planar interface at the focus.

TABLE II

Subjects ANd Body Sites Studied in this WORK.

\begin{tabular}{ccccl}
\hline Transducer & $\begin{array}{c}\text { Number of } \\
\text { Subjects }\end{array}$ & $\begin{array}{c}\text { Age } \\
\text { Mean (SD) }\end{array}$ & $\begin{array}{c}\text { Number of } \\
\text { Data sets }\end{array}$ & Body sites \\
\hline I (PI50) & $14(7$ male, 7 female) & $36(14.3)$ & 22 & $\begin{array}{l}\text { Forearm dorsal, } 6 \\
\text { forearm volar, } 9 ; \text { thigh, } 5 ; \\
\text { fingertip, } 2\end{array}$ \\
II (PI75) & $2(2$ male) & 31,47 & 2 & Forearm dorsal, 2 \\
III (PI3005) & 7 (3 male, 4 female) & $38(12.3)$ & 7 & $\begin{array}{l}\text { Forearm dorsal, } 1 ; \\
\text { forearm volar, } 6\end{array}$ \\
\hline
\end{tabular}

${ }^{1}$ For transducer II, the actual ages for subjects are given.

dermatological applications. Transducer II on the other hand had poorer lateral resolution and hence only two subjects were studied to demonstrate the methods. A total of 31 data sets were studied.

\section{Estimation of System Functions}

For each transducer, the axial and lateral system functions were estimated using one subject using the higherorder spectral method and were subsequently used for the deconvolution for all data sets obtained using that transducer. Both the axial and lateral pulses were estimated on the RF data corresponding to the dermis, after suitable correction for the attenuation and diffraction effects. For the axial pulse, about 200 echo lines of 128 samples each were used (i.e., 200 averages were used to compute the mean third order cumulants). A similar number of waveforms were used for determining the lateral point spread function. Fig. 3 shows the estimated axial and lateral profiles for transducer I. It can be seen that the estimated axial pulse and the DB14 wavelet in Fig. 1 are quite similar. Also, the DB6 wavelet has the closest match to the lateral point spread function of all the Daubechies wavelet functions.

\section{Deconvolution Results}

Fig. 4 shows an example of an image before and after deconvolution obtained using transducer $\mathrm{I}$. It can be seen that the image after deconvolution is able to more clearly show hypoechoic structures in the thigh, presumably hair follicles. It should be noted that the number of samples per echo line is usually much larger than the number of echo lines; therefore, the data were resized to maintain the same scale in the axial and lateral directions before displaying the images. This resizing tends to offset the resolution improvement in the images. In order to quantify the improvement in resolution due to the deconvolution, the $-6 \mathrm{~dB}$ correlation lengths of the RF data corresponding to the images were computed before and after deconvolution (before image resizing). Similar measures have been used previously by other researchers [3], [7]. It also should be pointed out that the $-6 \mathrm{~dB}$ correlation length is not the same as the axial and lateral resolutions mentioned in Table $I$. The resolution gain was defined as the ratio of the $-6 \mathrm{~dB}$ correlation lengths of the RF data before deconvolution to that after deconvolution (before any resizing was done for display purposes). For this data set, the axial and lateral resolution gains were 1.88 and 3.65 , respectively.

Fig. 5 shows an image of the fingertip region obtained on a 31-year-old male subject using transducer I. After deconvolution, the fingerprints on the surface appear more continuous rather than broken as in the original image. The axial and lateral resolution gains for this data set were 2.35 and 4.92 , respectively.

Fig. 6 shows an image of the forearm skin of a 31-yearold male subject obtained using transducer II. For trans- 

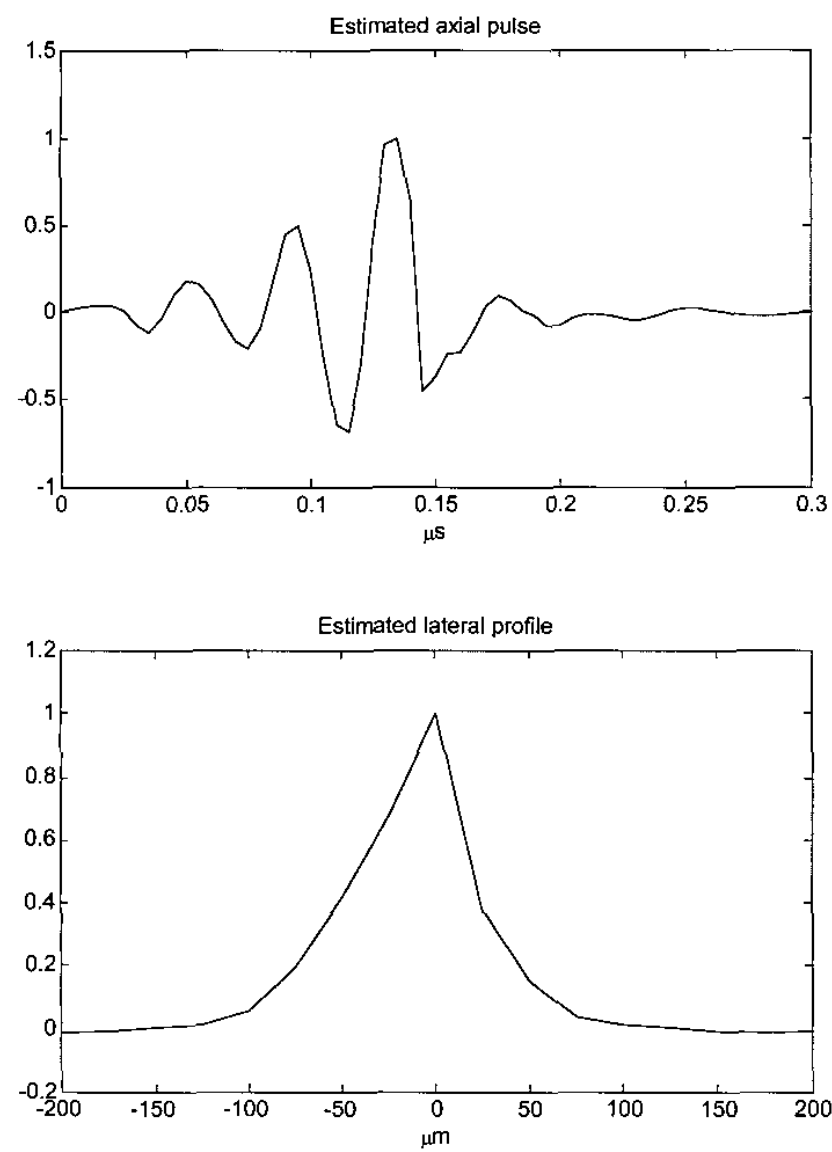

Fig. 3. Estimated axial and lateral system functions for transducer I.

ducer II, the original lateral resolution was poorer than that of transducer I due to its larger f-number. Therefore, the improvement in resolution is more clearly seen in this case. The axial and lateral resolution improvements were 5.4 and 14.8 , respectively.

Table III shows the results for all data sets studied in this work. The improvements in axial and lateral resolution were better for transducer II, which is expected as its original lateral resolution was the poorest to begin with. Transducer III had better axial and lateral resolution for the original images due to its higher center frequency and larger bandwidths; therefore, the improvement was smaller than those of the other two transducers. The improvement in axial resolution also is proportional to the improvement in lateral resolution, indicating that the axial and lateral directions are not truly uncorrelated.

\section{Discussion And Conclusions}

This paper presents a robust method for deconvolution of high-frequency ultrasound skin images using a combination of higher-order spectral methods and wavelet analysis. These methods also are applicable to ultrasonic images of other tissues. For the general deconvolution problem in which the system function also is unknown, a unique solution for both the system function $h(n)$ and the tissue signal $x(n)$ does not exist. For example, two trivial solutions that satisfy the deconvolution problem in the absence of noise are $h(n)=\delta(n), x(n)=y(n)$ and $h(n)=y(n)$, $x(n)=\delta(n)$. Thus, some assumptions regarding the system function and/or the tissue signal needs to be in order to obtain a mcaningful solution. One such assumption is that the system function $h(n)$ and tissue function $x(n)$ have different cepstral signatures and can be separated [2]. A further assumption that the system function is minimum phase simplifies the procedure as the complex cepstrum can be computed from a knowledge of the real cepstrum and phase unwrapping problems are avoided [5], [21]. Bayesian deconvolution, which uses a stochastic; approach, is another method for image restoration. Commonly this method is implemented assuming a Gaussian model for the probability density function of tissue reflectivity [4]. In our work we assumed that the tissue signal $x(n)$ was non-Gaussian, which was supported by our earlier results $[10]$. Therefore, we used higher-order spectral methods to extract the pulse. An advantage of this method is that the tissue signal and the pulse signal easily can be decoupled. Therefore, a pulse or lateral point spread function estimated from one data set could be used for all subsequent data sets, making the procedure simple and efficient. This was demonstrated in this work, where only one pulse was used for all subjects and meaningful resultis were obtained.

Taxt [6] has compared seven different deconvolution methods that use cepstral computations and found that first order cepstral methods might have an advantage over higher order methods for conventional 1-10 $\mathrm{MHz}$ ultrasound. However, even in that work, data from liver and gastric antrum showed good results with higher order methods, presumably because the echo signals were nonGaussian. In our earlier work we showed that envclope signals collected from the transducers used in this work: from skin tissues are non-Rayleigh; therefore, the corresponding RF signals are non-Gaussian. In particular we have used third-order methods, which require the additional assumption of nonzero third-order cumulants. Phys. ical reasons for such assumptions were given by Abeyratne et al. [22]. The tissue is modeled as a collection of diffuse, mixed, and resolvable periodic scatterers, and nonGaussianity arises due to the mixed and periodic resolvable components. Third-order methods were used because they are computationally simpler than the fourth or even higher-order methods.

To the best of our knowledge, all previous deconvolution methods in ultrasound have used a simple inverse Wiener filtering after pulse estimation. In the present work, we have used a wavelet-based approach to the inverse Wiener filtering to estimate the tissue signals. Our experience thus far is that the wavelet-Wiener method is better than the simple Wiener method. A useful side comment is that wavelet-based methods are more natural in pulse-echo ultrasound analysis in general as a specific wavelet could be easily chosen to very efficiently represent the signal. Therefore, tasks such as filtering, denoising, and compres- 
(a) Original image

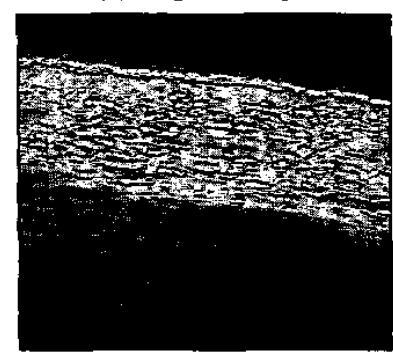

(c) Axial correlation

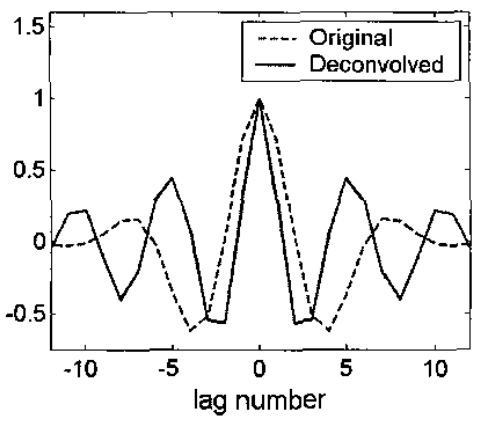

(b) Deconvolved image

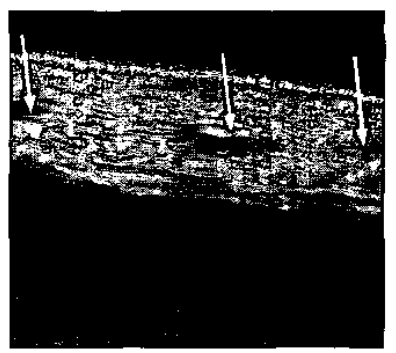

(d) Lateral correlation

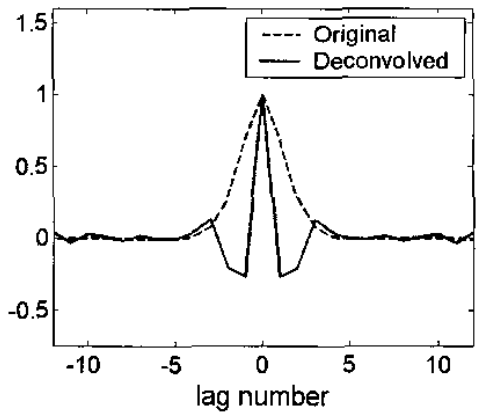

Fig. 4. (a) Image of the skin at the thigh region of a 30-year-old male subject obtained using transducer I before deconvolution. The field of view is $5 \mathrm{~mm}$ wide by $4.5 \mathrm{~mm}$. (b) The same image after axial and lateral deconvolution. The hypoechoic structures (arrow marks) are possibly hair follicles, and they are better displayed in the deconvolved image than in the original image. Panels (c) and (d) show the axial and lateral correlation functions of the images, respectively. The correlation functions after deconvolution have smaller main lobe widths than those of the original image.

(a) Original image

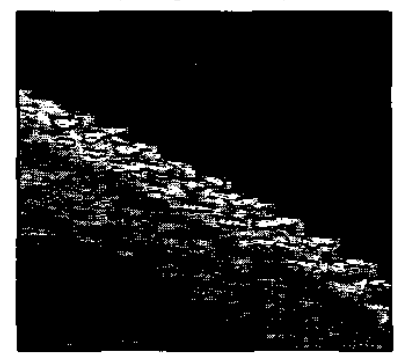

(c) Axial correlation

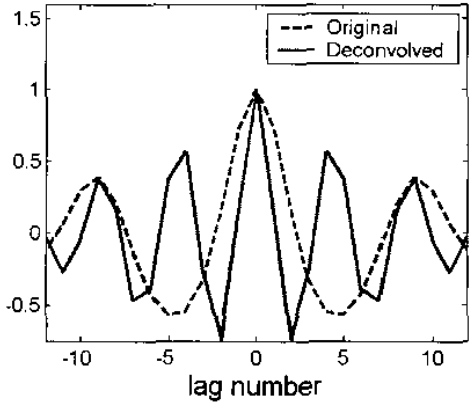

(b) Deconvolved image

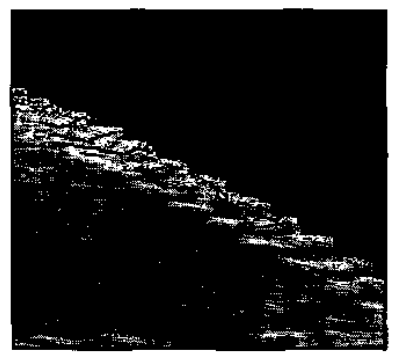

(d) Lateral correlation

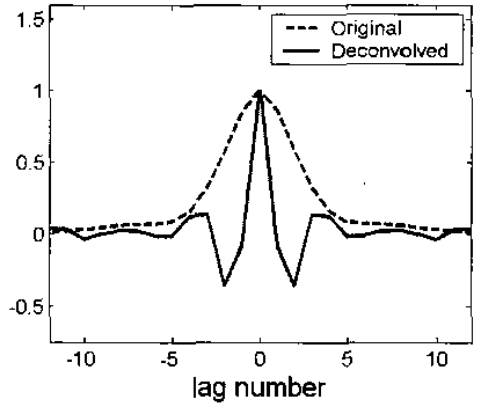

Fig. 5. (a) Image of a fingertip skin of a 31-year-old male subject obtained using transducer I before deconvolution. The field of view is $5 \mathrm{~mm}$ wide by $4.5 \mathrm{~mm}$ deep. (b) The same image after axial and lateral deconvolution. The fingerprints on the surface appear better in the deconvolved image in that they appear more continuous. Panels (c) and (d) show the axial and lateral correlation functions of the images, respectively. The correlation functions after deconvolution have smaller main lobe widths than those of the original image. 
(a) Original image

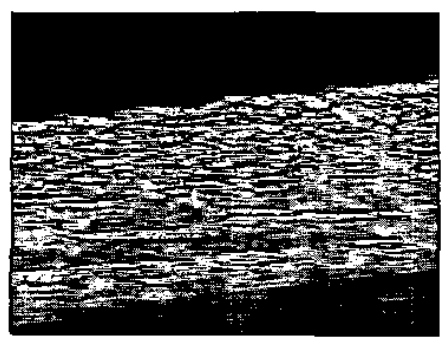

(c) Axial correlation

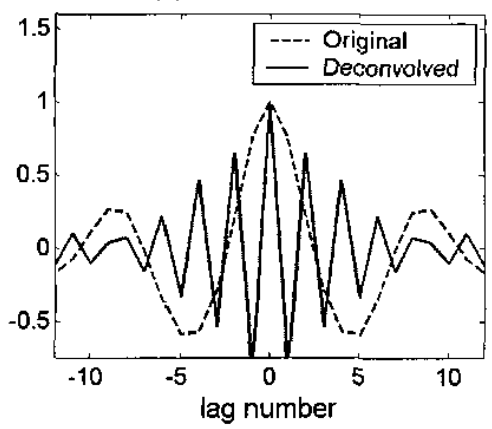

(b) Deconvolved image

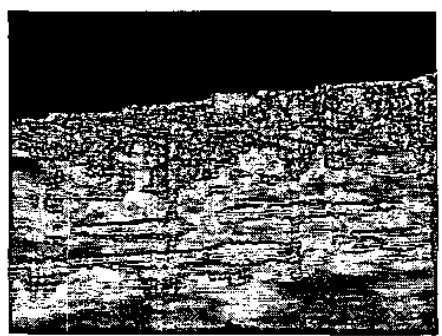

(d) Lateral correlation

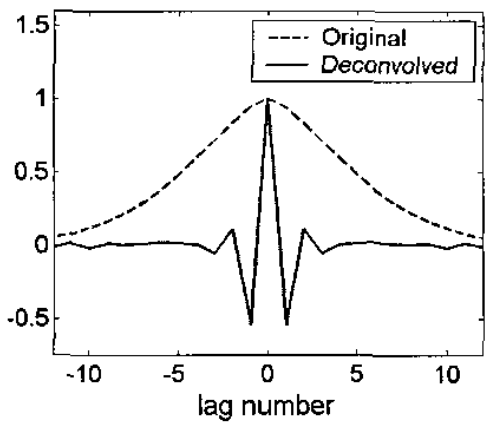

Fig. 6. (a) Image of the skin at the forearm region of a 31-year-old male subject obtained using transducer II before deconvolution. The field of view is $6 \mathrm{~mm}$ wide by $4.5 \mathrm{~mm}$. (b) The same image after axial and lateral deconvolution. The improvement in resolution in the deconvolved image can be seen. Panels (c) and (d) show the axial and lateral correlation functions of the images, respectively. The correlation function 3 after deconvolution have smaller main lobe widths than those of the original image.

TABLE III

Resolution Gains Obtained Due to Deconvolution. ${ }^{1}$

\begin{tabular}{cccc}
\hline Transducer & $\begin{array}{c}\text { Number of } \\
\text { data sets }\end{array}$ & $\begin{array}{c}\text { Axial resolution gain } \\
\text { (mean } \pm \text { SD) }\end{array}$ & $\begin{array}{c}\text { Lateral resolution gain } \\
\text { (mean } \pm \mathrm{SD})\end{array}$ \\
\hline I (PI50) & 22 & $1.99 \pm 0.34$ & $4.01 \pm 0.88$ \\
II (PI75) & 2 & $4.1,5.4$ & $11.1,14.8$ \\
III (PI3005) & 7 & $1.21 \pm 0.39$ & $2.76 \pm 0.51$ \\
\hline
\end{tabular}

${ }^{1}$ The results are based on $-6 \mathrm{~dB}$ correlation lengths in the axial and lateral directions of the RF signal.

${ }^{2}$ For transducer II, the actual values are shown.

sion could be very efficiently accomplished using wavelet representations.

In this work the axial and lateral deconvolution methods were decoupled for the sake of simplicity. For a given tissue, the finest resolution possible in the axial direction depends on the sampling frequency. The resolution improvement in the case of transducer II was higher even in the axial direction compared to those of the other two transducers, even though the same sampling rate was used for all transducers. This is most likely because the axial and lateral signals are not fully decoupled and each Ascan is not strictly a representation of one line of data. Therefore, in future studies, full 2-D deconvolution methods using wavelet-Wiener methods might be more useful.

The deconvolution model in (1) is a simplified one in that other effects such as phase aberrations have not been accounted for. In the field of astronomy in which deconvoIution methods have been used successfully, phase aberration correction (using adaptive optics) is often done before deconvolution. Such a procedure is not currently possible with high-frequency ultrasound as phased-array transducers are not available yet; but, with the development of such systems in the future [23], it may be possible to combine phase-aberration correction and deconvolution to significantly improve the quality of high-frequency ultrasound images.

\section{REFERENCES}

[1] J. A. Jensen, J. Mathorne, T. Gravesen, and B. Stage, "Deconvolution of in-vivo ultrasound B-mode images," Ultrason. Imag., vol. 15, pp. 122-133, 1993. 
[2] T. Taxt, "Restoration of medical ultrasound image using twodimensional homomorphic deconvolution," IEEE Trans. Ultrason., Ferroelect., Freq. Contr., vol. 42, pp. 543-554, 1995.

[3] U. R. Abeyratne, A. P. Petropulu, and J. M. Reid, "Higher order spectra based deconvolution of ultrasound images," IEEE Trans. Ultrason., Ferroelect., Freq. Contr., vol. 42, pp. 1064-1075, 1995.

[4] O. Husby, T. Lie, T. Lango, J. Hokland, and H. Ruc, "Bayesian 2-D deconvolution: A model for diffuse ultrasound scatter ing," IEEE Trans. Ultrason., Ferroelect., Freq. Contr., vol. 48, pp. $121-130,2001$.

[5] D. Adam and O. Michailovich, "Blind deconvolution of ultrasound sequences using nonparametric local polynomial estimates of the pulse," IEEE Trans. Biomed. Eng., vol. 49, pp. 118-131, 2002.

[6] T. Taxt, "Comparison of cepstrum-based methods for radial blind deconvolution of ultrasound images." IEEE Trans. Ultrason., Ferroelect., Freq. Contr., vol. 44, pp. 666-674, 1997.

[7] U. R. Abeyratne, A. P. Petropulu, J. M. Reid, T. Golas, E. Conant, and F. Forsberg, "Higher order versus second order statistics in ultrasound image deconvolution," IEEE Trans. Ultrason., Ferroelect., Freq. Contr., vol. 44, pp. 1409-1416, 1997.

[8] J. M. Mendel, "Tutorial on higher-order statistics (spectra) in signal processing and system theory: Theoretical results and some applications," Proc. IEEE, vol. 79. pp. 278-305, 1991.

[9] S. El Gammal, C. El Gammal, K. Kasper, C. Pieck, P. Altmeyer, M. Vogt, and H. Ermert, "Sonography of the skin at $100 \mathrm{MHz}$ enables in vivo visualization of stratum corneum and viable epidermis in palmar skin and psoriatic plaques," J. Invest. Dermatol., vol. 113, pp. 821-829, 1999.

[10] B. I. Raju and M. A. Srinivasan, "Statistics of envelope of high frequency ultrasonic backscatter from human skin in vivo," IEEE Trans. Ultrason., Ferroelect., Freq. Contr., vol. 49, pp. $871-882,2002$.

[11] R. Neelamani, H. Choi, and R. G. Baraniuk, "Wavelet-based deconvolution for ill-conditioned systems," in IEEE Conf. Acoust. Speech, and Signal Processing (ICASSP), vol. 6, pp. 3241-3244, 1999.

[12] C. L. Nikias and J. M. Mendel, "Signal processing with higherorder spectra," IEEE Signal Processing Mag., vol. 10, pp. 10-37, 1993.

[13] R. Pan and C. L. Nikias, "The complex cepstrum of higher order cumulants and nonminimum phase system indentification," IEEE Trans. Acoust. Speech Signal Processing, vol. 36 pp. $186-205,1988$

[14] S. Mallat, A Wavelet Tour of Signal Processing. Boston: Academic, 1999.

[15] D. L. Donoho and I. M. Johnstone, "Ideal spatial adaptation by wavelet shrinkage," Biometrika, vol, 81, pp. 425-455, 1994.

[16] B. I. Raju and M. A. Srinivasan, "High frequency ultrasonic attenuation and backscatter cocfficients of in vivo normal human dermis and subcutancous fat," Ultrasound Med. Biol, vol. 27, pp. 1543-1556, 2001.

[17] X. Chen, D. Phillips, K. Q. Schwartz, J. G. Mottley, and K. J. Parker, "The measurement of backscatter coefficient from a broadband pulsc-ccho system: A new formulation," IEEE Trans. Ultrason., Ferroelect., Freq. Contr., vol. 44, pp. 515-525, 1997.

[18] D. L. Donoho, "Nonlinear solution of linear inverse problems by wavelet-vaguelette decomposition," Appl. Comput. Harmonic Anal, vol. 2, pp. 101-126, 1995

[19] A. D. Hillary and R. T. Chin, "Iterative Wiener filters for image restoration," IEEE Trans. Signal Processing, vol. 39, pp. 18921899,1991

[20] H. Choi and R. Baraniuk, "Analysis of wavelet-domain Wiener filters," in Proc. IEEE-SP Int. Symp. Time-Frequency and Time-Scale Analysis, 1998, pp. 613-616.

[21] J. A. Jensen and S. Leeman, "Nonparametric estimation of ultrasound pulses," IEEE Trans. Biomed. Eng., vol. 41, pp. 929-936, 1994.

[22] U. R. Abeyratne, A. P. Petropulu, and J. M. Reid, "On modeling the tissue response from ultrasound B-scan images," IEEE Trans. Med. Imag., vol. 15, pp. 479-490, 1996.

[23] T. K. Ritter, T. R. Shrout, R. Tutwiler, and K. K. Shung, "A $30-\mathrm{MHz}$ piezo-composite ultrasound array for medical imaging applications," IEEE Trans. Ultrason., Ferroelect., Freq. Contr., vol. 49 , pp. $217-230,2002$.

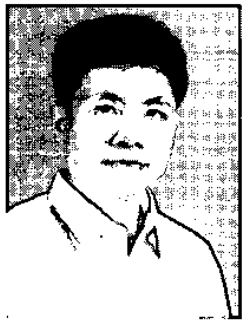

Suiren Wan received the M.S. degree in electrical engineering from the Southeast University, Nanjing, China, in 1982, and the Ph.D. degree from the Beijing Institute of Vacuum Electronic Technology, Beijing, China, in 1986.

Since 1993, he has been a Professor and Director of the Laboratory of Medical Electronics, Department of Biology and Biomedical Engineering, Southeast University. His research interests lie in the areas of computerized medical instruments and medical applications of electromagnetic and sound waves. From 2000 to 2003 he was a visiting professor in the Laboratory for Human and Machine Haptics, Research Laboratory of Electronics at MIT, Cambridge, $\mathrm{MA}$, working on signal processing using higher order spectra and wavelets.

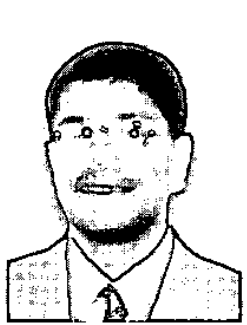

Balasundar I. Raju was born in Madras, India, in 1972. He received the bachelor's degree in mechanical engineering from the Indian Institute of Technology, Madras, in 1994. He received two master's degrees in 1998 , one in mechanical engineering and the other in electrical engineering and computer science, and a Ph.D. degree in electrical engineering and computer science in 2002, all from MIT, Cambridge, MA. He has spent two summers in the industry working on linear and nonlinear ultrasonic wave propagation problems. $\mathrm{He}$ is currently a senior member of the research staff at Philips Research, Briarcliff Manor, NY.

His research interests include high-frequency ultrasound, wave propagation, signal and image processing, skin imaging and characterization, and skin biomechanics.

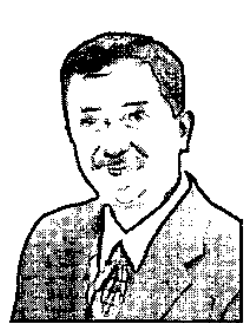

Mandayam A. Srinivasan received the bachelor's degree in civil engineering from Bangalore University, Bangalore, India, the master's degree in aeronautical engineering from the Indian Institute of Science, Bangalore, and the Ph.D. degree in applied mechanics from Yale University, New Haven, CT. He worked as a member of the research staff at the Department of Anesthesiology, Yale University School of Medicine, where he conducted research on the mechanics and mechanisms of the primate sense of touch. Since 1987 he has been at the Massachusetts Institute of Technology, Cambridge, MA, where he founded the Laboratory for Human and Machine Haptics, informally known as the Touch Lab (http://touchlab.mit.edu). He is currently the Director of the Touch Lab and a Senior Research Scientist with the Department of Mechanical Engineering and the Research Laboratory of Electronics at MIT.

Dr. Srinivasan has conducted pionecring investigations in skin biomechanics, tactile neuroscience, sensorimotor psychophysics, haptic device design, and multimodal rendering algorithms. He has demonstrated novel applications of this multidisciplinary research in areas such as the development of multiuser haptics in shared virtual environments, medical simulations involving force feedback for training, and direct control of machines from primate brain signals. As part of the research on human haptics, he has worked on a variety of techniques for imaging the skin, including video-microscopy, MRI, optical coherence tomography, and high-frequency uitrasound.

He has served as a member of the National Academy of Sciences committee on virtual reality and teleoperator systems, the MIT Virtual Environment and Teleoperation Research Consortium and the editorial board of the MIT Press journal PRESENCE: Teleoperators and Virtual Environments. He is also a member of the Society for Neuroscience. His work has been featured in print, radio, and television media around the world in news articles and programs focused on cutting-edge research in information technology and its future prospects. 